\title{
Low-energy vibrational excitations in polymethylmetacrylate with IR and RAMAN spectroscopy
}

\begin{abstract}
Based on IR and Raman spectroscopy data, it was determined the minimum size of a chain segment (torsional-vibrational segment) and we found that low-energy vibrational excitations in polymethylmethacrylate (PMMA) and its oligomers with a degree of polymerization $n=2,7,9$ and 50 are due to the low-angle correlated torsionalvibrational dynamics preparing the relaxation mobility of macromolecules.
\end{abstract}

Keywords: raman spectroscopy, chain segment, low-energy vibrational excitations, polymethylmethacrylate, relaxation mobility, macromolecules
Volume 3 Issue 3 - 2019

VA Ryzhov
loffe Physicotechnical Institute, Russian Academy Science, Russia

Correspondence: VA Ryzhov, loffe Physicotechnical Institute, Russian Academy Science, 19402I St. Petersburg, Russia, Tel 89313354279 , Email v.ryzhov@mail.ioffe.ru

Received: April 24, 2019 | Published: May 16, 2019

\section{Introduction}

As it is well known, depending mainly on the time-temperature scale at which they are studied, amorphous polymers exhibit viscoelastic behavior which involves different molecular motions, with very different dynamics. At low temperatures or short times, the molecular mobility is generally considered as localized and leads to a macroscopic mechanical or dielectric behavior, so called secondary relaxations. These motions are often described as rotations of lateral groups or as crankshaft motions of main chain segments. Though much work has already been published on the subject, the origin of these relaxations is still uncertain. In most currently manifestation molecular mobility in glassy polymers associated with low-energy excitation of the type: a) low-angular libration of monomer units of chain or molecules; ${ }^{1}$ b) correlated vibrations, manifesting as a boson peak (BP) in the IR and Raman spectra, ${ }^{2}$ and c) reorientation processes, manifesting as fast relaxation phenomena. ${ }^{3}$ The nature of $\mathrm{BP}$ is currently widely discussed. A number of authors believe that the appearance of additional quasi-local vibrational modes is associated with the existence of medium-order and cohesive inhomogeneity in disordered media. ${ }^{4}$ Others relate them to correlated vibrations: for example, in quartz- torsional vibrations in a chain of several $\mathrm{SiO} 4$ tetrahedra. ${ }^{5}$ In a previous paper ${ }^{6}$ Based on our comparative study of IR and Raman spectra of these low-energy excitation plasticized polymethylmethacrylate (PMMA) were presumably attributed to the librational motion of the polymer chain segment the size in several monomer units. The purpose of this paper is to experimentally test this assumption.

\section{IR and Raman spectroscopy}

IR and Raman spectroscopy have ample opportunities to solve a wide range of complex problems. Both techniques are completely complementary providing characteristic fundamental vibrations that are extensively used for the determination and identification of molecular structure. Although some vibrations may be active in both Raman and IR, these two forms of spectroscopy arise from different processes and different selection rules. In general, Raman spectroscopy is best at symmetric vibrations of non-polar groups while IR spectroscopy is best at the asymmetric vibrations of polar groups. Infrared and Raman spectroscopy involve the study of the interaction of radiation with molecular vibrations but differs in the manner in which photon energy is transferred to the molecule by changing its vibrational state. IR spectroscopy measures transitions between molecular vibrational energy levels as a result of the absorption of IR radiation. This interaction between light and matter is a resonance condition involving the electric dipole mediated transition between vibrational energy levels. Raman spectroscopy is a two-photon inelastic light-scattering event. Here, the incident photon is of much greater energy than the vibrational quantum energy, and loses part of its energy to the molecular vibration with the remaining energy scattered as a photon with reduced frequency. In the case of Raman spectroscopy, the interaction between light and matter is an off-resonance condition involving the Raman polarizability of the molecule. The IR and Raman vibrational bands are characterized by their frequency (energy), intensity (polar character or polarizability), and band shape (environment of bonds). Since the vibrational energy levels are unique to each molecule, the IR and Raman spectrum provide a "fingerprint" of a particular molecule. The frequencies of these molecular vibrations depend on the masses of the atoms, their geometric arrangement, and the strength of their chemical bonds. The spectra provide information on molecular structure, dynamics, and environment.

Low-frequency IR and Raman studies in the $400-10 \mathrm{~cm}^{-1}$ region constitute only a small part of molecular spectroscopy but they demonstrate their unique possibilities for investigating polymers. IR and Raman spectroscopy is traditionally applied for investigation of the high-frequency group of vibrations situated in a spectral region where only the main valences are deformed; these vibrations are purely intramolecular and, therefore, not influenced by changes in the polymer system. In contrast, most of the low-frequency vibrations are intermolecular, which means that they consist of collective excitations and are determined by the packing of chains in the lattice. The problems of the lattice and quasi-lattice vibrations, sensitivity to the conformational state of a chain and anharmonicity of vibrations, intrinsic vibrations of hydrogen bonds, the motion of heavy ions in ionomers and coulombic interactions have been widely discussed and they all look attractive for future investigations. For polymers, the lowfrequency region is of crucial interest because there the relaxational type of spectrum and the resonant type coexist. Special attention was 
given to the way how information about molecular motions giving rise to $\beta$-, $\gamma$ - and $\delta$-transitions in glassy polymers can be obtained from far IR and Raman spectra in addition to the other experimental data, e.g. by differential scanning calorimetry. The experimental evidence of the mechanism of $\delta$-relaxation, as a hindered rotation (libration) of monomeric units, as well as of $\beta$-relaxation as a reorientational motion of the chain part (close to the magnitude of the statistical segment) surmounting primarily the intermolecular potential barriers are essential. The main characteristics of these transitions were found to be related to main molecular parameters of polymers such as the structure of the monomeric unit, thermodynamic chain rigidity, cohesion energy, and the potential barrier to internal rotation. The discussions in this review stress the usefulness of the low-frequency IR and Raman spectra for the study of the chemical and physical structure and for the elucidation of the molecular dynamics in polymeric materials. We are sure that many more applications will appear as the instrumental techniques are improved and our theoretical understanding increases.

\section{Materials and methods}

Samples of amorphous PMMA and its short-chain oligomers with a degree of polymerization $n=2,7,9$, and 50 were synthesized at the Institute of Chemical Physics RAS using catalytic chain transfer to the cobalt porphyrin molecule. ${ }^{7}$ All of them, except for the dimer, were prepared in the form of films with a thickness of 10 to $100 \mu \mathrm{m}$ for recording the IR spectra and in the form of parallelepipeds with polished edges for obtaining Raman spectra. Dimer was placed in a cuvette with windows of poly-4-methyl-1-pentene, transparent at low frequencies. The measurements were carried out at two temperatures: room temperature and liquid nitrogen temperature. The IR spectra were recorded on two spectrometers: in the range from 10 to $50 \mathrm{~cm}^{-1}$ at the spectrometer of the Leningrad State University, ${ }^{8}$ upgraded with an OAP-7 receiver and a new filtration system, and in the range 50 $130 \mathrm{~cm}^{-1}$ using an FIS-21 Hitachi spectrometer. The resolution was $1-2 \mathrm{~cm}^{-1}$. The accuracy of determining the position of the maximum of the bands was 2-3 $\mathrm{cm}^{-1}$. The measurement error of the absorption coefficient $\mathrm{k}=\ln \left(\mathrm{J}_{0} / \mathrm{J}\right) /\left(\mathrm{t}-\mathrm{t}_{0}\right)$, where $\mathrm{J}_{0}$ and $\mathrm{J}$ are the transmittances of samples with a thickness of $t_{0}$ and $t$, respectively, was 5-10\%. Raman spectra were recorded on a Ramalog-5 double monochromator in a $90^{\circ}$ scattering geometry. The spectra were exited with the $488 \mathrm{~nm}$ line of an argon laser (the radiation power was $0.1 \mathrm{~W}$. The spectral width of the slit was $2 \mathrm{~cm}^{-1}$. The scattering intensity spectra I (v), averaged over ten scans in the region of $5-130 \mathrm{~cm}^{-1}$, were normalized taking into account the spectral function of the instrument and the frequency scattering factor. The calculation of low-frequency Raman spectra, based on the obtained experimental spectra, was carried out by converting the latter according to the formula widely used in the literature: ${ }^{9,10}$

$$
I_{R}(v)=I(v)\left[1-\exp \left(-\hbar v / k_{B} T\right)\right]=I(v)[N(v)+1]-1, \text { where } N(v)=\left[\exp \left(\hbar v / k_{B} T\right)-1\right]-1-\text { Bose factor }
$$

The representation of the Raman spectrum in the coordinates $I(v) /[N(v)+1]$ has the advantage that it can significantly reduce the contributions of the elastic (Rayleigh) and relaxation components of the scattering wing. In addition, the recalculated spectra are directly comparable with the spectra of dielectric loss $\varepsilon$ "(v). Since at optical frequencies the absorption coefficient $\mathrm{k}$ is proportional to $v \cdot \varepsilon$ " $(v)$, the spectrum $I(v) /[N(v)+1]$ corresponds to IR -spectrum in $\mathrm{k} / v$ coordinates. The Raman and IR spectra thus recalculated can be correctly compared with each other.

\section{Results and discussions}

Figure 1 shows the Raman and IR spectra of PMMAand its oligomers in the range of $10-150 \mathrm{~cm}^{-1}$ in the coordinates $I R=I(v) /(N(v)+1)$ and $\mathrm{k} / \mathrm{v}$, respectively. They have the typical for low-frequency spectra of linear amorphous polymers the form of a broad asymmetric band with a maximum at $80-90 \mathrm{~cm}^{-1}$ and a shoulder at the frequency of the boson peak at $15-20 \mathrm{~cm}^{-1}$. The inset shows their experimental Raman and Fir- spectra at the same frequencies. The arrows indicate the position of the boson peak in the spectra. Earlier studies of lowfrequency IR spectra of low-molecular substances and polymers make it possible to attribute the considered band to absorption due to the libration (rotational vibrations) of monomeric units in PMMA macromolecules, that is, to the absorption by the Poley mechanism. ${ }^{11}$ To the same vibrations should be assigned and the discussed band in Raman spectrum of PMMA, having a comparable contour and close frequency position of the maximum. ${ }^{12}$ A slight broadening of the band and a shift of its maximum towards low frequencies in the spectra

of oligomers are apparently caused by an increase in the amplitude and anharmonicity of the librational vibrations in low-molecular analogs of PMMA. The identity of the mechanisms of band formation in the low-frequency IR and Raman spectra of condensed media is confirmed by numerous data. ${ }^{13}$ In one of the simplest models used to analyze absorption by the Poley mechanism, ${ }^{14}$ the libration motion of a molecule with the moment of inertia (I) takes place within the potential well formed by its nearest environment, having the form $U(\phi)=U 0 \sin 2 \pi \phi / 2 \xi$, circular frequency

$$
\omega=2 \pi c v l i b r \pi / \xi\left(U_{0} / 2 I\right)^{1 / 2},
$$

Where $U_{0}$ is the potential well depth $\xi$ is its half-angle aperture (width at a height equal to half the full barrier), $\phi$ is the amplitude of libration. The model predicts the position of the maximum of the libration band in the low-frequency spectra of polar and non-polar liquids. The intermolecular barriers of the libration motion found with its help correspond to those obtained by other methods. ${ }^{15}$ A similar approach for polymers gives good agreement with the experiment, provided that the monomer unit is the librator. In such calculations, it is assumed that the librator has the shape of a ball with the moment of inertia $\mathrm{I}=2 \mathrm{MR}^{2} / 5$, where $\mathrm{M}$ is its molecular weight and $\mathrm{R}$ is the equivalent radius. In the case of PMMA, estimating the size of a librator by $v_{\text {libr }}=95 \mathrm{~cm}^{-1}$ in expression (1), starting from the activation energy of the $\delta$ transition $^{16}$ and the presence of limited torsional vibrations in macromolecules with an amplitude of $10-15^{0},{ }^{17}$ gives $\mathrm{R}=0.25 \mathrm{~nm}$, which is close to the van der Waals radius of the monomeric unit of this polymer. 
The result obtained is an additional argument in favor of assigning a band with a maximum at $95 \mathrm{~cm}^{-1}$ to low-frequency Raman and IR- spectra of PMMA and its oligomers to the libration movement of the monomeric unit of the macromolecule. Let us turn to another feature of the low-frequency IR and Raman spectra of PMMA and its oligomers - the shoulder on the low-frequency wing of the studied band. In the initial (experimental) Raman spectrum of the PMMA, the frequency of the maximum of the boson peak $v_{\mathrm{BP}}$ is $16-18 \mathrm{~cm}^{-}$ ${ }_{1.9,18}$ After reduction the experimental spectrum to the coordinates $I(v) / . N(v)+1$, the boson peak transforms into a shoulder on the low-frequency wing of the band at the same frequency, $v_{B P}$ (Figure 1A). When comparing reduced Raman spectra with the Fir-spectra given to the $\mathrm{k}(v) / v$ coordinates (Figure 1B), it can be seen that and in the reduced Fir-spectra PMMA a similar shoulder is also observed at the boson peak frequency $v_{\mathrm{BP}} \approx 16-20 \mathrm{~cm}^{-1}$. In order to clarify the molecular nature of the in the low-frequency wing of the libration band, we consider the reduced IR and Raman spectra of PMMA and its oligomers (Figure 1) \& (Figure 2). It follows from them that with an increase in the length of the chain, the shoulder on the spectral curve shifts towards lower frequencies. The dependence of its position $-v_{\mathrm{BP}}$ on the length of the oligomer chain, expressed by the number of monomer units $n$, is presented in Figure 3. The frequency of the main maximum of the band due to the libration of the monomeric unit also falls on this dependence. This confirms that the shoulder on the low-frequency wing of the main band also characterizes the libration motion, which, as the $v_{\mathrm{BP}}$ dependence on $n$ shows, should be attributed to a motion in which several monomer units are involved. The graph in Figure 3 allows us to estimate the maximum number of chain links involved in this correlated libration movement. In the case of PMMA and its oligomers, it does not exceed 5-7 monomers. The length $\mathrm{L}$ of such a segment of the main chain can also be determined using the following expression: ${ }^{9}$

$$
L \approx c t(c \cdot v B P)^{-1}
$$

Where $\mathrm{c}$ is the speed of light in vacuum, $c_{t}$ is the "transverse" speed of sound (shear wave velocity). For PMMA, $c_{t} \approx 1.42 \mathrm{~km} / \mathrm{s}^{18}$ Substituting this value and the frequency of the boson peak $v B P=17 \mathrm{~cm}^{-1}$ into (2), we find that in PMMA, $L \square 3 \mathrm{~nm}$. The obtained value corresponds to the length of the section of the polymer chain of six monomer units and is close to the size of the statistical Kuhn segment for PMMA. ${ }^{19}$ The statistical segment characterizes in this case the dynamic interconnection of neighboring monomers in the polymer chain.

It is noteworthy that the linear dimensions of the region of collective vibrational excitations and in other glassy polymers are comparable with the sizes of their statistical segments. Thus, from the analysis of the Raman spectra of glassy polystyrene (PS) and its oligomers, ${ }^{20,21}$ it follows that the size of this region here is $6-8$ monomer units (the statistical segment of PS is equal to eight links ${ }^{19}$ ). For polybutadiene ${ }^{21}$ and polypropylene glycol, ${ }^{22}$ the region of collective vibrational excitations includes 11 and 7 monomer units, respectively, which is also close to the values of the statistical segments for these polymers. If in expression (2) to substitute the value of the frequency of the maximum of the libration the band width $v=95 \mathrm{~cm}^{-1}$, then the found value of the length of the segment of the polymer molecule is $0.5 \mathrm{~nm}$, that is, the length of the monomer unit in PMMA.

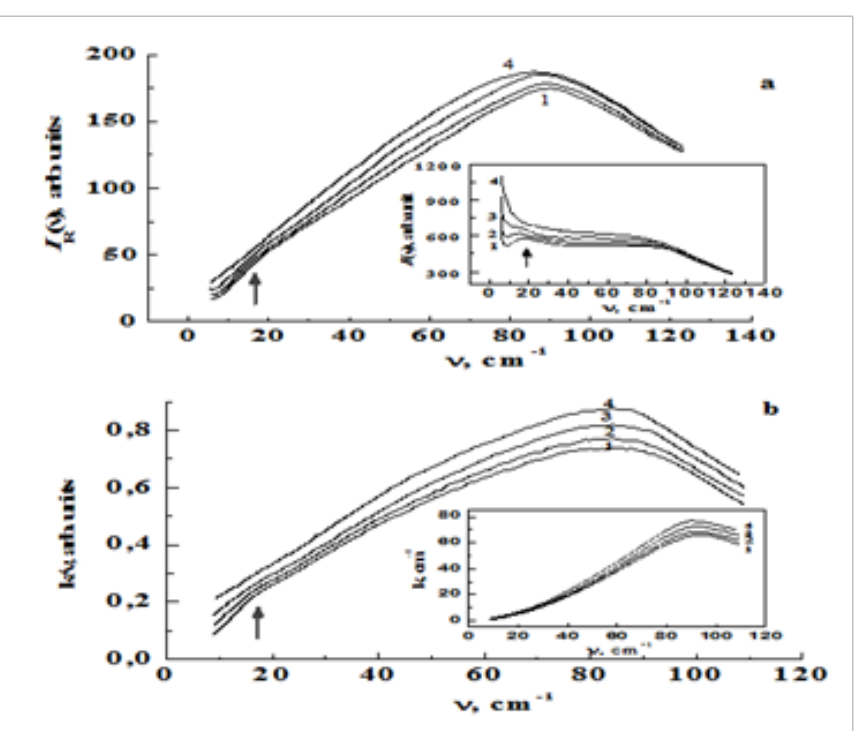

Figure I Reduced Raman (a) and IR spectra (b) in the range of $10-120 \mathrm{~cm}-I$ of PMMA (I) and its oligomers with $n=9$ (2), 7 (3), and 2 (4).

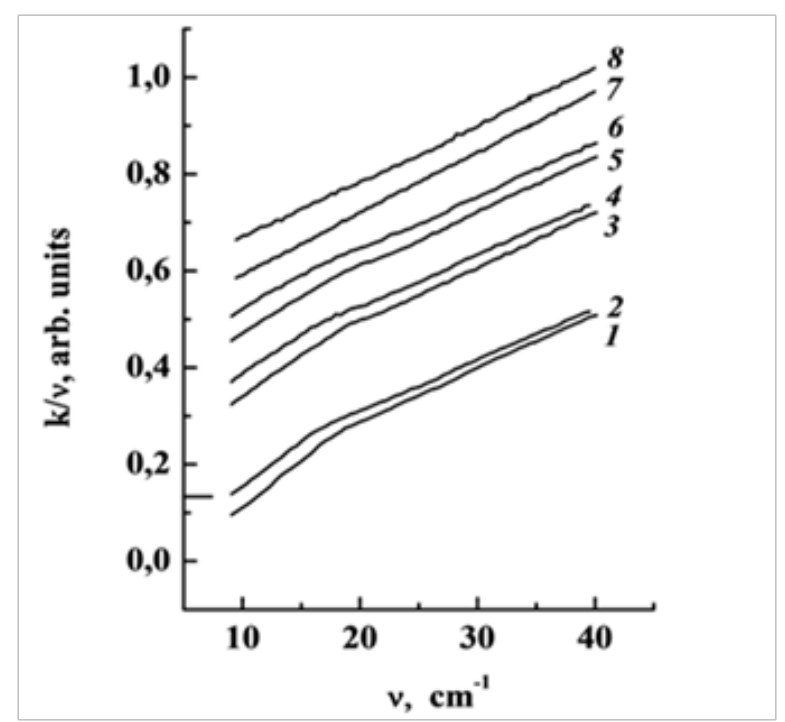

Figure 2 Reduced Fir-spectra of PMMA $(I, 2)$, oligomer with $n=9(3,4)$, oligomer with $n=7(5,6)$ and oligomer with $n=2(7,8)$. At $T=90(1,3,5.7)$ and $293 \mathrm{~K}(2,4,6,8)$ in the range of $10-40 \mathrm{~cm}^{-1}$. The spectra of oligomers are shifted along the ordinate axis.

So, a wide band in the low-frequency IR and Raman spectra of PMMA and its oligomers with a maximum at $95 \mathrm{~cm}^{-1}$ characterize the torsional vibrations of the monomeric unit of the polymer, whereas the shoulder on its low-frequency wing at $16-18 \mathrm{~cm}^{-1}$ is due to correlated torsional vibrations involving several monomeric units. It is significant that the linear size of the region of correlated libration excitations found from the $v_{\mathrm{BP}}$ frequency in the low-frequency IR and Raman spectra corresponds to the length of the statistical segment - the minimal fragment of the polymer chain necessary for a rotational isomeric transition in it. The movement of fragments of such length, which takes place in the places of the least dense 
packing of macromolecules, underlies the $\beta$-relaxation. ${ }^{19,23}$ It is an analogue of the Johari-Goldstein relaxation ${ }^{23}$ in low molecular weight vitrified liquids, which consists in the reorientation of the molecule under the action of thermal fluctuations. In this case, the molecule overcomes the potential barrier $\mathrm{U}_{0}$ formed by its nearest environment. The $\beta$-transition is preceded by a universal $\gamma$-process: ${ }^{23}$ Small-angle torsional vibrations - molecular libration, which, as already noted, causes the appearance of the Poley band in the low-frequency IR and Raman spectra of condensed media at the frequency $v_{\text {libre }}$. That is, using low-frequency IR and Raman spectra, one can directly determine both the parameters of the libration movement preparing $\beta$-relaxation and the sizes of the parts of the polymer chain involved in this movement during the $\beta$-transition. Turn again to Figure 2 . It can be seen from it that the intensity of the low-frequency wing of the libration band (in the frequency range below $v_{\mathrm{BP}}$ ) increases with increasing temperature. A similar effect was previously observed in the Raman spectra of glassy solids. ${ }^{9}$ According to these studies, the increase in intensity below $v_{\mathrm{BP}}$ is due to the increasing contribution of the $\beta$-process with temperature. Consequently, the frequency of the bend in the lowfrequency IR and Raman spectra of PMMA corresponds; in addition, to the transition boundary from resonance absorption to relaxation, when the number of monomers involved in the libration movement is sufficient for conformational rearrangement in the polymer chain.

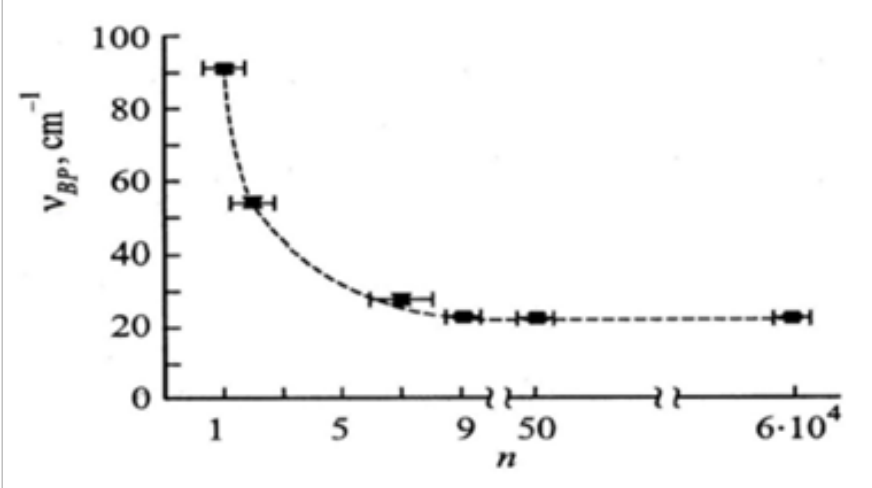

Figure 3The dependence of $v_{\mathrm{BP}}$ on the degree of polymerization, expressed the number of monomer units $n$.

\section{Conclusion}

Thus, analysis of the low-frequency IR and Raman spectra of PMMA and its oligomers showed the following.

The studied band - the Poley band - refer to the librations of the monomer unit, which determine the universal $\gamma$-process ( $\delta$-transition in polymer terminology). The maximum length of the correlation section of the macromolecule found from the frequency $v_{B P}$ of the shoulder on the low-frequency wing of the studied band is close to the size of the statistical segment, the movement of which underlies the relaxation $\beta$ - transition. Frequencies lower than the frequency of the boson peak $v_{B P}$ correspond to the relaxation mobility of macromolecules, which is realized when the number of monomer units involved in the libration movement becomes sufficient to effect a conformational transition in the polymer chain.

\section{Acknowledgments}

None.

\section{Conflicts of interest}

The author declares there is no conflict of interest.

\section{References}

1. VA Bershtein, VA Ryzhov. Far infrared spectroscopy of polymers. Adv Polym Sci. 1994;114:43-121.

2. VA Ryzhov, VA Bershtein. Low-frequency librational vibrations, boson peak, and interchain interactions in a vitreous polymer. Phys Solid State. 2008;50:1985.

3. VA Ryzhov. Study of fast relaxation in a glassy polymer by far-IR spectroscopy. Phys Solid State. 2017;59(7):1450-1453.

4. R Shuker, RW Gammon. Raman-Scattering Selection-Rule Breaking and the Density of States in Amorphous Materials. Phys Rev Letts. 1970;25:222.

5. SD Bembenek, BB Laird. Instantaneous normal modes analysis of amorphous and supercooled silica. J Chem Phys. 2001;114(5):2340.

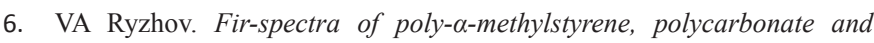
polymethylmethacrylate oligomers. In Physics and Chemistry of Polymers. Tver state un-t. 2003;9:37.

7. BR Smirnov, IS Morozova, AP Marchenko, Dokl Akad Nauk SSSR. 1980;255:608

8. VA Ryzhov, MV Tonkov. In Molecular Spectroscopy Leningr. State unit. 1973;2:103.

9. VN Novikov, AP Sokolov, B Stube, et al. Connection between quasielastic Raman scattering and free volume in polymeric glasses and supercooled liquids. J Chem Phys. 1997;107(4):1057.

10. G Caliskan, A Kisliuk, VN Novikov, et al. Relaxation spectra in poly (methylmethacrylate): Comparison of acoustic attenuation and light scattering data. J Chem Phys. 2001;114(22):10189.

11. J Ph Poley. Microwave dispersion of some polar liquids. Appl Sci Res Sec B. 1955;4(1):337-387.

12. JF Viras, TA King. Low frequency excitation in amorphous acrylic polymers. Polymer. 1984;25(7):899-905.

13. VS Libov, TS Perova. The infrared (IR) and Raman spectra of vitreous poly (methyl methacrylate) (PMMA). Tr Gos Opt Inst. 1992;81:215.

14. IW Larkin. Simple models of the far infra-red absorption of polar molecules in liquid and rotator phases. Chem Soc Faraday Trans II. 1973;69:1278-1290.

15. MW Evans, GJ Evans, WT Coffey, et al. Molecular Dynamics and Theory of Band Spectra. Wiley, New York. 1982.

16. VA Ryzhov, VA Bershtein. New binaphthyl-containing liquid-crystalline copolymers forming a chiral nematic phase. Vysokomol Soedin Ser A. 1989;31:451.

17. TM Birshtein, OB Ptitsyn. Konformatsia makromolecul. Nauka, Moscow. 1964.

18. AP Sokolov, A Kisliuk, M Soltwisch, et al. Medium-range order in glasses: Comparison of Raman and diffraction measurements. Phys Rev Lett. 1992;69:1540.

19. VA Bershtein, VM Egorov. Differential Scanning Calorimetry in the Physical Chemistry of Polymers. Khimiya, Leningrad. 1990.

20. RJ Spells, I. W Shepherd. Concentration dependence of the zero-field splittings of triplet phenanthrene- $d_{10}$ oriented in biphenyl single crystals. $J$ Chem Phys. 1977;66(4):1427. 
21. U Buchenau, C Pecharreman, R Zorn, et al. Neutron Scattering Evidence for Localized Soft Modes in Amorphous Polymers. Phys Rev Lett. 1996;77:659.

22. R Bergman, C Svanberg, D Andersson, et al. Relaxational and vibrational dynamics of poly(propylene glycol). J Non-Cryst Solids. 1998;235237:225-228

23. Yu Ya Gotlib, AA DarinskiÏ, Yu E Svetlov. Physical Kinetics of Macromolecules. Khimiya, Leningrad. 1986.
24. GP Johari, M Goldstein. Viscous Liquids and the Glass Transition. III. Secondary Relaxations in Aliphatic Alcohols and Other Nonrigid Molecules. J Chem Phys. 1971;55(9):4245.

25. CJ Reid, MW Evans. Dielectric and far infrared study of solutions in the glassy state from $100 \mathrm{~Hz}$ to $10 \mathrm{THz}$ : Discovery and characterization of the universal $\gamma$ process. J Chem Phys. 1982;76(5):2576.

26. NV Surovtsev, JA Wiedersich, VN Novikov, et al. Light-scattering spectra of fast relaxation in glasses. Phys Rev B. 1998;58:14888. 